\title{
A Systematic Review on Intra-Abdominal Pressure in Severely Burned Patients
}

Steven G Strang ${ }^{1}$, Esther M.M. Van Lieshout ${ }^{1}$, Roelf S. Breederveld ${ }^{2}$, Oscar J.F. Van Waes ${ }^{1}$

${ }^{1}$ Erasmus MC, University Medical Center Rotterdam, Department of Surgery-Traumatology, Rotterdam, The Netherlands

${ }^{2}$ Red Cross Hospital, Burn Unit, Beverwijk, the Netherlands

\section{Correspondence:}

Oscar J.F. Van Waes, MD

Erasmus MC, University Medical Center Rotterdam

Dept. of Surgery-Traumatology

Room H-822k

P.O. Box 2040

3000 CA Rotterdam

The Netherlands

Phone: +31.10.7031050

Fax: +31.10 .7032396$

E-mail: o.vanwaes@erasmusmc.nl 


\section{Abstract}

Objective: Intra-abdominal hypertension (IAH) and abdominal compartment syndrome (ACS) are complications that may occur in severe burn patients. Evidenced based medicine for these patients is in its early development. The aim of this study was to provide an overview of literature regarding IAH and ACS in severe burn patients.

Methods: A systematic search was performed in Cochrane Central Register of Controlled Trials, PubMed, Embase, Web of Science and CINAHL on October 1, 2012. These databases were searched on 'burn', ‘intra-abdominal hypertension', ‘abdominal compartment syndrome', synonyms and abbreviations. Studies reporting original data on mortality, abdominal decompression or abdominal pressure related complications were included. Results: Fifty publications met the criteria, reporting 1616 patients. The prevalence of ACS and IAH in severe burn patients is $4.1-16.6 \%$ and $64.7-74.5 \%$, respectively. The mean mortality rate for ACS in burn patients is $74.8 \%$. The use of plasma and hypertonic lactated resuscitation may prevent IAH or ACS. Despite colloids decrease resuscitation volume needs, no benefit in preventing IAH was proven. Escharotomy, peritoneal catheter drainage, and decompression laparotomy are effective intra-abdominal pressure (IAP) diminishing treatments in burn patients. Markers for IAP-related organ damage might be superior to IAP measurement itself.

Conclusion: ACS and IAH are frequently seen devastating complications in already severely injured burn patients. Prevention is challenging but can be achieved by improving fluid resuscitation strategies. Surgical decompression measures are effective and often unavoidable. Timing is essential since decompression should prevent progression to ACS rather than limit its effects. Prognosis of ACS remains poor, but options for care improvement are available in literature. 


\section{Introduction}

Severely injured burn patients are at risk for elevated intra-abdominal pressure (IAP). The World Society of Abdominal Compartment Syndrome (WSACS) defines burn injury as an independent risk factor for abdominal compartment syndrome (ACS). ACS is a syndrome of new organ failure resulting from a sustained or repeated IAP $>20 \mathrm{mmHg}$ (1). A sustained or repeated IAP $\geq 12 \mathrm{mmHg}$ without signs of organ failure is termed Intra-Abdominal Hypertension (IAH). IAH and ACS are detrimental complications in the critically ill, even more in severely burned patients. However, evidenced based medicine for these severely injured patients is still in its early development.

Greenhalgh et al. were the first to describe the occurrence and effects of elevated IAP among four cases of burn injury in children in 1994 (2). In a prospective analysis of 30 severe burn patients, they demonstrated that an IAP $\geq 30 \mathrm{mmHg}$ is associated with a 3 to 4 times increased sepsis and mortality rates. This publication initiated an increase of awareness of IAP-related complications and its devastating effects. It took until 1999 before Ivy et al. (3) reported on intra-abdominal hypertension (IAH) and abdominal compartment syndrome (ACS) in adult burn patients. IAH and ACS are diagnosed using various IAP measuring techniques, measurements can be continuous and direct or indirect intra-vesical; this last method is included in the guidelines of WSACS and is accurate in burn patients as well (4-6)

IAH and ACS result from large fluid resuscitation volumes in combination with severe systemic inflammatory response syndrome (SIRS) (7). Both resuscitation volume and SIRS are dependent of the burn injury severity (8). Even though the most commonly used ParklandBaxter formula states a use of $4 \mathrm{~mL} / \mathrm{kg} / \%$ of (burned) total body surface area (TBSA), larger volumes are often given $(9,10)$. This may lead to a phenomenon called 'fluid creep' which gives rise to excessive edema formation and 'third spacing' of the fluid excess (11). This is swift process; intra-abdominal edema and ascites leading to IAH can emerge within only a 
few hours after sustaining the burn injury (12). SIRS in these patients becomes a selfperpetuating process caused by accumulation of pro-inflammatory cytokines in the resulting ascites fluid (13).

The second main factor that leads to IAH in burn patients are compliance decreasing burns of the abdominal or thoracic wall. The compliance curve of a healthy human abdomen shows it can easily contain 3L extra volume without a significant increase in IAP (14). When local burn injury is present, the abdomal volume capacity is smaller. Truncal burn and increased intra-abdominal volume can raise IAP independently, but when an inauspicious combination of these conditions occurs, patients can deteriorate fast. In that case, IAP can be relieved rapidly by longitudinal skin incision (escharotomy) of the truncal burns or eschars $(15,16)$.

ACS related new organ failure typically presents itself as oliguria or ventilation difficulties. They result from the body's inability to compensate and overcome the intraabdominal pressures which gives rise to tissue ischemia. Compensatory ability is strongly patient dependent, therefore measuring IAP alone is not sufficient in determining the patient's threat. Abdominal perfusion pressure (APP) defined as the mean arterial pressure (MAP) minus the IAP, is more suitable measurement $(1,17)$. To restore adequate perfusion pressures in ACS patients, decompression is needed. Early ACS recognition is of decisive importance for prompting such a decompression. Even when early recognition is achieved, ACS has a poor prognosis. Mortality rates of $44 \%$ up to $100 \%$ are reported for burn patients with ACS $(18,19)$.

Even though IAP-related complications in severe burn patients are dangerous, they occur quite common. Prevention of IAH and ACS should receive high priority in severe burn patients. Unfortunately resuscitation of burn patients with respect to IAH and ACS is based on limited evidence $(9,20,21)$. In order to identify the treatment elements that improve 
outcome for these patients, a concise overview of available evidence is needed. The aim of this systematic review was to provide a detailed overview of literature regarding epidemiology, therapy, and outcome of rising IAP related complications in major burn patients. 


\section{Methods}

A systematic search was conducted in Cochrane Central Register of Controlled Trials, PubMed, Embase, Web of Science and CINAHL on October 1, 2012. Databases were searched for the following terms: "burn” and synonyms combined with “abdominal compartment syndrome” or “intra-abdominal hypertension” and synonyms or abbreviations (Table 1). Reference lists of all manuscripts were reviewed to identify additional literature. Articles were screened on [title] and [abstract] for the exclusion criteria; no burn in combination with IAH or ACS, reviews, comments, animal studies, and questionnaire surveys. When no full-text was available after several attempts and when a double population was suspected, manuscripts were excluded too. The remaining articles were screened in full text and included when original patient data were present. No language criterion was used.

The level of evidence was determined according to Mahid et al. (22). Data regarding risk factors, diagnosis, treatment and outcome of these studies were extracted; conclusions of individual studies are discussed. 


\section{Results}

The primary search resulted in 500 hits. After applying the inclusion and exclusion criteria, 50 manuscripts remained (Figure 1); 21 case-reports or case series and 29 cohort studies. Especially the first publications used a variety of definitions and cut points for intraabdominal hypertension and abdominal compartment syndrome. Since WSACS stated unambiguous definitions for IAH and ACS in 2006, literature became more uniform and comparable (1). The heterogeneity in study populations and collected data and the lack of details on the IAP measurement techniques across studies made pooled analysis impossible.

\section{Prevalence}

By approximation, IAH prevalence according to WSACS guidelines ( $\geq 12 \mathrm{mmHg}$ ) is 64.7 $74.5 \%$ among patients with $\geq 20 \%$ of TBSA burned or having inhalation injury (Table 2). The prevalence of ACS among patients with $\geq 15 \%$ TBSA burned ranged from $4.1 \%$ to $16.6 \%$ (Table 3). Seven of nine manuscripts reported ACS rates between $4 \%$ and $16.6 \%$ independent of their variety in cutoff burn size.

\section{$\underline{\text { Outcome }}$}

In 21 case reports and series, a mortality rate of 50\% among 58 burn ACS patients was seen (2, 3, 5, 12, 16, 23-38) (Table 4). Nine cohort studies ( $\mathrm{N}=132$ total) report mortality rates between $44 \%-100 \%$, with a weighted average of $74.8 \%(18,19,39-45)$ (Table 5). No improving trend in mortality can be demonstrated over recent years. 


\section{$\underline{\text { Risk factors }}$}

Several obvious risk factors for ACS in burn patients exist. Decreased abdominal wall compliance (caused by local burns) is generally considered to contribute to the development of IAH. However, truncal burns are not a prerequisite per se for IAH in burn patients (3). Resuscitation volume is the second risk factor for IAH. A small prospective case series of nine patients showed that fluid resuscitation volume of $0.25 \mathrm{~L} / \mathrm{kg}$ in the early post burn period resulted in an IAP of $24.4 \mathrm{mmHg}$ (27). Exceeding this volume (also described as 'Ivy score'), is a suggested independent risk factor for developing IAH/ACS. Oda et al. noted that a resuscitation volume larger $0.3 \mathrm{~L} / \mathrm{kg}$ in the first $24 \mathrm{~h}$ post burn were at risk for ACS (46). Greenhalgh et al. described sepsis, oliguria, hypoventilation and hypotension as specific signs for IAH or ACS, apart from sepsis evidence was insufficient to determine whether these risk factors are independent $(1,2)$. Additionally, injury severity (in \% TBSA burned), seems linearly associated with ACS incidence (47). A burn size of $\geq 40 \%$ TBSA proved to be an independent risk factor for ACS (25). Electrical etiology of burn injury is another possible risk factor; in a matched case control study the ACS prevalence in electrical burns patients was $4 \%$ vs. $1.5 \%$ in thermally injured patients (48). Nonetheless sample size was too small to reach statistical significance. The last risk factor is skeletal immaturity; in a retrospective study of 1014 patients, six out of 10 cases that developed ACS (mean TBSA burned $72 \%$ ) were non-adults with a mean age of six years (41).

\section{$\underline{\text { Resuscitation }}$}

Large volumes of resuscitation fluid are needed to maintain appropriate hemodynamics in severely burned patients. However, excessive fluid resuscitation may increase the IAP level in burn patients $(2,3,27)$. Similarly, pediatric burn patient prove to be at risk for excessive chylous ascites accumulation as a result of large resuscitation fluid volumes leading to ACS 
(35). A multivariable linear regression analysis of 72 burn patients (mean TBSA burned 44.5\%), showed percentage TBSA burned, age, weight, and intubation before admission to a burn center to significantly influence fluid requirements in the first 24 hours after burn injury (49).

Implementation of standardized military volume limiting burn resuscitation guidelines resulted in a decrease of a composite endpoint of ACS and mortality (50). Decreasing resuscitation volume of the Parkland formula from 4 to $3 \mathrm{ml} / \mathrm{kg} / \%$ TBSA is proposed in a case report of a pediatric burn patient who developed ACS and died as a result of 'fluid creep' (36). The use of a modified Brooke formula (2ml/kg/\%TBSA) proved to be a good alternative as well (51). A statistically significant reduction of resuscitation volumes $(\mathrm{p}=0.005)$ and peak IAP ( $\mathrm{p}=0.0001$ ) was achieved by using plasma instead of crystalloid resuscitation fluid (52). In a randomized trial, a statistically significantly lower resuscitation volume $(\mathrm{p}<0.01)$ and IAP after 24h $(\mathrm{p}<0.05)$ was demonstrated in severe burn patients without inhalation injury after resuscitation with hypertonic lactated saline (HLS) compared lactated Ringer’s (53). Using colloids to reduce resuscitation volumes was not associated with worse outcome in burn patients when compared with the standard Parkland formula (54).

\section{$\underline{\text { Decompression laparotomy }}$}

Decompression laparotomy is the most used and accepted abdominal decompressive measure. Even in children this is an adequate therapy without specified adverse effects (19). Although indications for decompression laparotomy are usually straight forward, some nuances can be made. Since burn patients have already lost the protective barrier of normal skin, there is diversion in opinion whether decompression laparotomy might induce unacceptable morbidity. Although hemodynamic parameters in burn patients rapidly improve after decompression laparotomy, it does not decrease rates of acute lung injury and multi organ 
dysfunction syndrome (MODS) (55). Acute lung injury and multi organ dysfunction syndrome may be more severe after decompression laparotomy than before $(\mathrm{p}<0.05$ and $\mathrm{p}<0.01$, respectively) and more severe than in similar severely injured burn patients without IAH ( $p<0.05$ and $p<0.05$, respectively). Similarly, open abdominal decompression is associated with higher mortality rates among patients aged 80 years or older (81\%) than among younger patients (30-50\%) (56). If possible, surgeons should consider avoiding decompression laparotomy in fragile patient categories.

Alternative IAP lowering techniques which can be applied prior to decompression laparotomy include escharotomy of circumferential abdominal burn wounds, percutaneous catheter decompression, bowel care, nasogastric tube decompression, flushing the bladder catheter to ensure patency, pharmacologic paralysis, and sedation (41).

\section{Escharotomy}

When large burns of the trunk are present, abdominal and thoracic wall escharotomy is the appropriate early decompressive measure. Thoracoabdominal escharotomy is mostly performed in a standardized pattern; an incision in the anterior axillary line bilaterally, one along the lower margin of the rib cage and two symmetric longitudinal cuts at the anterior abdomen. A small cohort study proved abdominal escharotomy to decrease mean bladder pressure significantly in low grade IAH $(\mathrm{p}<0.001)(16)$. Another 8-patients cohort study demonstrated that escharotomy statistical significantly reduces IAP from 38 to $19 \mathrm{mmHg}$ $(\mathrm{p}<0.01)$ and results in a direct improvement of cardiovascular parameters in high grades of IAH (57). Patients of both studies presented themselves within 2-6 hours after burn injury and developed IAH requiring decompressive escharotomy within 24 hours after the injury. This endorses the need for early IAP measurement in standard burn care, especially when burn injury of the trunk is present. 


\section{$\underline{\text { Percutaneous catheter decompression }}$}

IAH in burn patients can occur as a result of accumulation of ascites and bowel edema. Percutaneous catheter decompression decompresses the abdomen by releasing the ascites without influencing the edema. When ascites is present, placement of a peritoneal dialysis catheter or angiocatheter (depending on patient size) and leaving it sutured in place is generally sufficient. In a small study, this minimally invasive measure decreased the IAP by $14 \mathrm{mmHg}$, with a rapid improve in hemodynamic parameters (26). A 13-patient cohort study concluded percutaneous catheter decompression to be effective in patients with $<80 \%$ TBSA burned with concomitant inhalation injury. More severely injured patients required decompression laparotomy and died (44). The effectiveness of percutaneous catheter decompression with respect to abdominal perfusion pressure (APP) was confirmed in a case report (32). Lastly, a retrospective case-control study determined percutaneous catheter decompression to be a safe decompression alternative (43). Nonetheless, when no signs of ascites are present, this technique is obsolete. Peritoneal catheter decompression is indicated when escharotomy has failed (40), beware of non-functioning catheters.

\section{$\underline{\text { Temporary abdominal closure }}$}

When faced with an open abdomen after decompression laparotomy, the question raises how to close it. Closing an open abdomen is especially difficult in patients with abdominal burns. In order to prevent complications of the open abdomen, temporary abdominal closure device can be used. Temporary abdominal closure devices again cause additional damage to the already injured abdominal wall and are associated with infectious complications such as abscess and fistula formation. In a six-patient cohort study, four burn patients died of sepsis with MODS after applying a vacuum-assisted TAC (42). 
To bridge the period unto definitive abdominal closure, visceral coverage should be preferred over vacuum assisted temporary abdominal closure devices until proven to be safe in burn patients. The possibility for early partial abdominal closure should be regularly assessed in order to prevent persistent dehiscence. The component separation technique of Ramirez is a primary closure technique which does not use foreign materials $(34,58)$. In a series of burn patients with ACS, two patients survived more than 30 days after abdominal closure with this technique (34). No intra-abdominal abscess and entero-cutaneous fistula formations were observed in this study.

The danger for ACS remains after temporal abdominal closure (23). Recurrent or tertiary ACS endorses the need for continued IAP monitoring after temporary closure

\section{Management}

IAH in burn patients is frequently a result of large resuscitation volumes, but this may lead to other complications such as acute ischemic optic neuropathy as well (33). This 'fluid creep' is a dangerous condition of which IAH (and ACS) is only a single expression. Acute kidney injury, a possible complication of IAH/ACS, does not help in draining this volume overload. Acute kidney injury is found in $40 \%$ of severely burned patients with ACS, and is associated with 50\% mortality rate (59). Burn patients with AKI require dialysis, which is associated with high mortality rates as well. Renal deterioration in burn patients can be reduced by diminishing the use of nephrotoxic medication (60). If IAH emerges, one should be aware of this other severe non-surgical or systemic complications. These complex multi-system complications require a multi-disciplinary approach, in which intensivists, surgeons, anesthesiologists, and nutritionists can play a crucial role in patient survival $(37,61,62)$. 


\section{Discussion}

This systematic review listed best literature regarding prevalence, treatment and outcome of IAH and ACS in severe burn patients, and provides more clarity regarding occurrence and mortality of ACS in burn patients. There are several shortcomings of the individual reports, the most obvious being the limited level of scientific evidence. Moreover, most papers do not mention clearly whether the diagnosis IAH was made based upon a one time "peak" measurement or on repeated sequential measurements. Nevertheless, some amendments can be made for this review as well. For example, the broad range of interest of this search aims at most important findings of the available literature regarding IAP-related complications in severe burn patients. Subsequently, minor details were not mentioned in this report. Much literature is available regarding burn care or ACS and IAH, but little and often poor evidence is found on the combination of conditions. More research is needed to decrease IAP related morbidity and mortality. A promising clue for this can be found in the following. Even though a large increase in use in abdominal decompressions is seen, no decrease in laparotomies for IAH related non-occlusive intestinal ischemia was seen over recent years (45). This ischemia induces massive inflammatory response which creates a vicious circle which indirectly leads to the development of ACS. Tools for early recognition of IAP related splanchnic ischemia are not available, but are probably more important than the measurement of IAP or APP itself $(19,38)$. 


\section{Conclusion}

Abdominal compartments syndrome and especially intra-abdominal hypertension are frequently seen devastating complications in the already severely injured burn patients. IAH prevalence is $64.7-74.5 \%$ among patients with $\geq 20 \%$ TBSA burned or with inhalation injury. ACS is seen in $4.1-16.6 \%$ of patients with $\geq 15 \%$ of TBSA burned. IAH or ACS can be prevented by decreasing resuscitation volume. Strict monitoring of urine output or hemodynamic parameters to prevent 'fluid creep' is of great importance. Using the modified Brooke formula, plasma, hypertonic lactated or colloid resuscitation is preferred over crystalloid use, especially in more severely injured, older and heavier patients intubated prior to admission on a burn unit.

Truncal burns in ACS patients require immediate escharotomy and should be followed by increasingly invasive decompression measures if no relieve is achieved. Timing of decompression is essential since it should rather prevent progression to abdominal compartment syndrome than limiting its effects.

Prognosis of abdominal compartments syndrome is very poor with a mean mortality rate of 74.8\%. IAH/ACS-burn patient outcome can be further improved by superior resuscitation regimes, better understanding of the inflammatory response after burn injury and tools for early recognition of splanchnic ischemia. 
Figure 1 - Flowchart of literature search

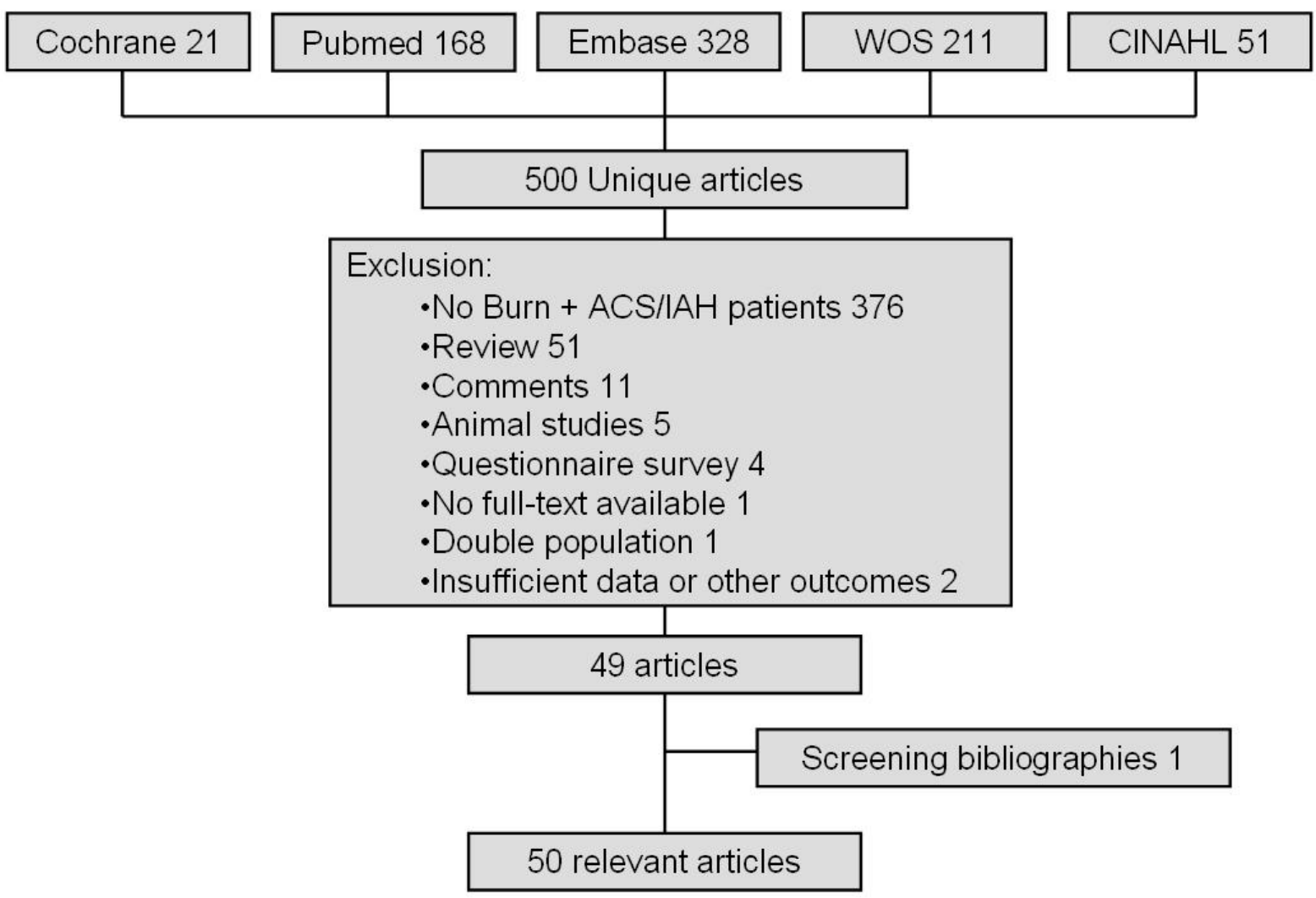


Table 1 - Search query

(burn OR burns OR burning OR burnings OR burned OR burnt OR scald OR scalds OR scalding OR scorch OR scorching OR singe OR singed OR blaze OR blazed OR “blast injury” OR “blast injuries”) AND (“abdominal compartment syndrome” OR ACS OR “abdominal compartment syndromes” OR “abdominal compartmental syndrome” OR “abdominal compartmental syndromes” OR “abdominal hypertension” OR “intra-abdominal hypertension” OR “IAH” OR “intra abdominal hypertension” OR “abdominal pressure” OR “intra-abdominal pressure” OR “intra abdominal pressure” OR “IAP”) 
Table 2 - Prevalence of IAH among burn patients

\begin{tabular}{lclllc}
\hline Author & LOE & Burn cutoff & IAH cutoff & N $=$ & Prevalence \\
\hline Oda et al. (2006) (53) & 2 & $\geq 40 \%$ TBSA & $\geq 22 \mathrm{mmHg}$ & 36 & $36 \% *$ \\
Sanchez et al. (2009) (63) & 5 & Mech. Vent. & $\geq 12 \mathrm{mmHg}$ & 33 & $64.7 \%$ \\
Malbrain et al (2010) (64) & 3 & Mech. Vent. & $\geq 12 \mathrm{mmHg}$ & 55 & $74.5 \%$ \\
\hline
\end{tabular}

*WSACS stated IAH cutoff pressure at $12 \mathrm{mmHg}$, therefore this number is of less relevance.

LOE, Level Of Evidence according to Mahid et al (22) 
Table 3 - Prevalence of ACS among burn patients

\begin{tabular}{lcccc}
\hline Author & LOE & Cutoff point & $\mathrm{N}=$ & Prevalence \\
\hline Hobson et al. (2002) (41) & 3 & 'Acute burn' & 1014 & $1 \%$ \\
Markel et al (2009) (47) & 3 & 'Acute burn' & 51 & $1.8 \%$ \\
Oda et al (2006) (46) & 3 & $\geq 40 \%$ TBSA & 48 & $16.6 \%$ \\
Ennis et al (2008) (50) & 3 & $\geq 30 \%$ TBSA & 118 & $11 \%$ \\
Mosier et al (2011) (61) & 3 & $\geq 20 \%$ TBSA & 153 & $4.6 \%$ \\
Klein et al (2007) (49) & 3 & $\geq 20 \%$ TBSA & 72 & $4.2 \%$ \\
Yenikomshian et al (2011) (62) & 3 & $\geq 20 \%$ TBSA & 50 & $8 \%$ \\
Cartotto et al (2010) (18) & 3 & $\geq 15 \%$ TBSA & 194 & $4.1 \%$ \\
Dulhunty et al (2008) (54) & 3 & $\geq 15 \%$ TBSA & 80 & $16 \%$ \\
\hline
\end{tabular}

Seven of nine cohort studies reported ACS prevalence of 4.1-16 \% among patients burned $\geq 15 \%$ TBSA

TBSA, Total Body Surface Area burned; LOE, Level Of Evidence according to Mahid et al (22) 
Table 4 - Mortality of severe burn patients with ACS in case descriptions

\begin{tabular}{lcc|lcc}
\hline Author & N & Mortality & Author & N & Mortality \\
\hline Greenhalgh et al (1994) (2) & 4 & $3(75 \%)$ & Ball et al (2006) (23) & 1 & $0(0 \%)$ \\
Ivy et al (1999) (3) & 3 & $3(100 \%)$ & Jensen et al (2006) (28) & 3 & $2(67 \%)$ \\
Ivy et al (2000)(27) & 10 & $2(20 \%)$ & Levis et al (2006) (30) & 4 & $3(75 \%)$ \\
Mayes et al (2000) (31) & 2 & $1(50 \%)$ & Parra et al (2006) (32) & 1 & $0(0 \%)$ \\
Corcos et al (2001) (26) & 3 & $2(67 \%)$ & Muangman et al (2007) (5) & & N/A \\
Wilson et al (2001) (38) & 1 & $0(0 \%)$ & Poulakidis et al (2009) (34) & 3 & $2(67 \%)$ \\
Blinderman et al (2002) (24) & 1 & $1(100 \%)$ & Thamm et al (2009) (37) & 1 & $0(0 \%)$ \\
Tsoutsos et al (2003) (16) & 10 & $4(40 \%)$ & Lamb et al (2010) (29) & & N/A \\
Pirson et al (2004) (33) & 1 & $0(0 \%)$ & Rogers et al (2010) (36) & 1 & $1(100 \%)$ \\
Britt et al (2005) (25) & 4 & $4(100 \%)$ & Rocourt et al (2011) (35) & 2 & $0(0 \%)$ \\
Rodas et al (2005) (12) & 1 & $0(0 \%)$ & Total & $\mathbf{5 6}$ & $\mathbf{2 8 ~ ( 5 0 \% )}$ \\
\hline
\end{tabular}


Table 5 - Mortality of severe burn patients with ACS in cohort studies

\begin{tabular}{lccc}
\hline Author & LOE & N & Mortality \\
\hline Hobson et al (2002) (41) & 3 & 10 & $6(60 \%)$ \\
Latenser et al (2002) (44) & 5 & 4 & $4(100 \%)$ \\
Hershberger et al (2007) (40) & 5 & 25 & $22(88 \%)$ \\
Chung JY et al (2007) (39) & 5 & 9 & $5(56 \%)$ \\
O’Mara et al (2007) (19) & 5 & 16 & $7(44 \%)$ \\
Latenser et al (2008) (43) & 4 & 9 & $4(44 \%)$ \\
Keremati et al (2008) (42) & 5 & 6 & $4(67 \%)$ \\
Cartotto et al (2010) (18) & 3 & 8 & $8(100 \%)$ \\
Van Niekerk et al (2010) (45) & 3 & 45 & $39(87 \%)$ \\
Total & & $\mathbf{1 3 2}$ & $\mathbf{7 4 . 8 \%}$ \\
\hline
\end{tabular}

LOE, level of evidence according to Mahid et al (22) 
1. Malbrain ML, Cheatham ML, Kirkpatrick A, et al: Results from the International Conference of Experts on Intra-abdominal Hypertension and Abdominal Compartment Syndrome. I. Definitions. 2006; 32(11):1722-1732.

2. Greenhalgh DG, Warden GD: The importance of intra-abdominal pressure measurements in burned children. 1994; 36(5):685-690.

3. Ivy ME, Possenti PP, Kepros J, et al: Abdominal compartment syndrome in patients with burns. 1999; 20(5):351-353.

4. World Society of the Abdominal Compartment Syndrome. Available at http://www.wsacs.org/consensus_summary.php; Accessed Dec. 1, 2012.

5. Muangman P, Muangman S, Suvanchote S, et al: Abdominal compartment syndrome monitoring in major burn patients with Siriraj device catheter. 2007; 90(2):384-390.

6. Waes OF, Jaquet J, Hop WJ, et al: A Single-Lumen Central Venous Catheter for Continuous and Direct Intra-abdominal Pressure Measurement. 2009; 35(6):532-537.

7. Kirkpatrick AW, Ball CG, Nickerson D, et al: Intraabdominal hypertension and the abdominal compartment syndrome in burn patients. 2009; 33(6):1142-1149.

8. $\quad$ Evers LH, Bhavsar D, Mailander P: The biology of burn injury. 2010; 19(9):777-783.

9. Tricklebank S: Modern trends in fluid therapy for burns. 2009; 35(6):757-767.

10. Baxter CRMD: Guidelines For Fluid Resuscitation. [Miscellaneous]. [Review]. 1981; 21(Supplement 8):687-689.

11. Saffle JI: The phenomenon of "fluid creep" in acute burn resuscitation. 2007; 28(3):382-395.

12. Rodas EB, Malhotra AK, Chhitwal R, et al: Hyperacute abdominal compartment syndrome: an unrecognized complication of massive intraoperative resuscitation for extra-abdominal injuries. 2005; 71(11):977-981.

13. Kowal-Vern A, Ortegel J, Bourdon P, et al: Elevated cytokine levels in peritoneal fluid from burned patients with intra-abdominal hypertension and abdominal compartment syndrome. 2006; 32(5):563-569.

14. Shay S, Schreiber M, Richter J: Compliance curves during peritoneal dialysate infusion are like a distensible tube and are similar at multiple UGI sites. 1999; 94(4):1034-1041.

15. Orgill DP, Piccolo N: Escharotomy and decompressive therapies in burns. 2009; 30(5):759-768.

16. Tsoutsos D, Rodopoulou S, Keramidas E, et al: Early escharotomy as a measure to reduce intraabdominal hypertension in full-thickness burns of the thoracic and abdominal area. 2003; 27(12):1323-1328.

17. Cheatham ML, White MW, Sagraves SG, et al: Abdominal perfusion pressure: a superior parameter in the assessment of intra-abdominal hypertension. 2000; 49(4):621-626; discussion 626-627.

18. Cartotto R, Zhou A: Fluid creep: the pendulum hasn't swung back yet! 2010; 31(4):551-558.

19. O'Mara MS, Reed N, Palmieri TL, et al: Immediate and long-term outcomes of decompressive laparotomy for abdominal compartment syndrome in pediatric burn patients... American Burn Association 39th Annual Meeting, March 20-23, 2007, Manchester Grand Hyatt, San Diego, California. 2007; 28(2):S137-S137.

20. Berger MM, Bernath MA, Chiolero RL: Resuscitation, anaesthesia and analgesia of the burned patient. 2001; 14(4):431-435. 
21. Azzopardi EA, McWilliams B, Iyer S, et al: Fluid resuscitation in adults with severe burns at risk of secondary abdominal compartment syndrome--an evidence based systematic review. 2009; 35(7):911-920.

22. Mahid SS, Hornung CA, Minor KS, et al: Systematic reviews and meta-analysis for the surgeon scientist. 2006; 93(11):1315-1324.

23. Ball CG, Kirkpatrick AW, Karmali S, et al: Tertiary abdominal compartment syndrome in the burn injured patient. 2006; 61(5):1271-1273.

24. Blinderman C, Lapid O, Shaked G: Abdominal compartment syndrome in a burn patient. 2002; 4(10):833-834.

25. Britt RC, Gannon T, Collins JN, et al: Secondary abdominal compartment syndrome: risk factors and outcomes. 2005; 71(11):982-985.

26. Corcos AC, Sherman HF: Percutaneous treatment of secondary abdominal compartment syndrome. 2001; 51(6):1062-1064.

27. Ivy ME, Atweh NA, Palmer J, et al: Intra-abdominal hypertension and abdominal compartment syndrome in burn patients. 2000; 49(3):387-391.

28. Jensen AR, Hughes WB, Grewal H: Secondary abdominal compartment syndrome in children with burns and trauma: a potentially lethal complication. 2006; 27(2):242246.

29. Lamb CM, Berry JE, DeMello WF, et al: Secondary abdominal compartment syndrome after military wounding. 2010; 156(2):102-103.

30. Levis C, Ali F: Significance of early diagnosis of abdominal compartment syndrome in major burns. 2006; 14(3):175-178.

31. Mayes T, Gottschlich MM, Warden GD: Nutrition intervention in pediatric patients with thermal injuries who require laparotomy. 2000; 21(5):451-456; discussion 450451.

32. Parra MW, Al-Khayat H, Smith HG, et al: Paracentesis for resuscitation-induced abdominal compartment syndrome: an alternative to decompressive laparotomy in the burn patient. 2006; 60(5):1119-1121.

33. Pirson J, Zizi M, Jacob E, et al: Acute ischemic optic neuropathy associated with an abdominal compartment syndrome in a burn patient. 2004; 30(5):491-494.

34. Poulakidas S, Kowal-Vern A: Component separation technique for abdominal wall reconstruction in burn patients with decompressive laparotomies. 2009; 67(6):14351438.

35. Rocourt DV, Hall M, Kenney BD, et al: Respiratory failure after pediatric scald injury. 2011; 46(9):1753-1758.

36. Rogers AD, Karpelowsky J, Millar AJ, et al: Fluid creep in major pediatric burns. 2010; 20(2):133-138.

37. Thamm OC, Leitsch S, Spanholtz TA, et al: Complicated course of a burned patient with severe inhalation injury: A case report. 2009; 1(4):224-227.

38. Wilson MD, Dziewulski P: Severe gastrointestinal haemorrhage and ischaemic necrosis of the small bowel in a child with $70 \%$ full-thickness burns: a case report. 2001; 27(7):763-766.

39. Chung JY, Latenser BA, Burke BA, et al: Pilot study: percutaneous drainage prevents abdominal compartment syndrome in significant thermal injury... American Burn Association 39th Annual Meeting, March 20-23, 2007, Manchester Grand Hyatt, San Diego, California. 2007; 28(2):S146-S146.

40. Hershberger RC, Hunt JL, Arnoldo BD, et al: Abdominal compartment syndrome in the severely burned patient. 2007; 28(5):708-714. 
41. Hobson KG, Young KM, Ciraulo A, et al: Release of abdominal compartment syndrome improves survival in patients with burn injury. 2002; 53(6):1129-1133; discussion 1133-1124.

42. Keramati M, Srivastava A, Sakabu S, et al: The Wittmann Patch(trademark) as a temporary abdominal closure device after decompressive celiotomy for abdominal compartment syndrome following burn. 2008; 34(4):493-497.

43. Latenser BA, Beins NT, Born JM, et al: Closed percutaneous drainage is definitive treatment of abdominal compartment syndrome in burn patients... Proceedings of the American Burn Association: 40th Annual Meeting, Tuesday through Friday, April 29 May 2, 2008, Hyatt Regency Chicago, Chicago, Illinois. 2008; 29(2):S112-S112.

44. Latenser BA, Kowal-Vern A, Kimball D, et al: A pilot study comparing percutaneous decompression with decompressive laparotomy for acute abdominal compartment syndrome in thermal injury. 2002; 23(3):190-195.

45. Van Niekerk W, Markell KW, Cancio L: Abdominal surgery in burn patients: The rise of abdominal compartment syndrome has not eclipsed dead gut. 2010; 36:S382.

46. Oda J, Yamashita K, Inoue T, et al: Resuscitation fluid volume and abdominal compartment syndrome in patients with major burns. 2006; 32(2):151-154.

47. Markell KW, Renz EM, White CE, et al: Abdominal complications after severe burns. 2009; 208(5):940-947; discussion 947-949.

48. Handschin AE, Vetter S, Jung FJ, et al: A case-matched controlled study on highvoltage electrical injuries vs thermal burns. 2009; 30(3):400-407.

49. Klein MB, Hayden D, Elson C, et al: The association between fluid administration and outcome following major burn: a multicenter study. 2007; 245(4):622-628.

50. Ennis JL, Chung KK, Renz EM, et al: Joint Theater Trauma System implementation of burn resuscitation guidelines improves outcomes in severely burned military casualties. 2008; 64(2 Suppl):S146-151; discussion S151-142.

51. Chung KK, Wolf SE, Cancio LC, et al: Resuscitation of severely burned military casualties: fluid begets more fluid. 2009; 67(1529-8809 (Electronic)):231-237.

52. O'Mara MS, Slater H, Goldfarb IW, et al: A prospective, randomized evaluation of intra-abdominal pressures with crystalloid and colloid resuscitation in burn patients. 2005; 58(5):1011-1018.

53. Oda J, Ueyama M, Yamashita K, et al: Hypertonic lactated saline resuscitation reduces the risk of abdominal compartment syndrome in severely burned patients. 2006; 60(1):64-71.

54. Dulhunty JM, Boots RJ, Rudd MJ, et al: Increased fluid resuscitation can lead to adverse outcomes in major-burn injured patients, but low mortality is achievable. 2008; 34(8):1090-1097.

55. Oda J, Yamashita K, Inoue T, et al: Acute lung injury and multiple organ dysfunction syndrome secondary to intra-abdominal hypertension and abdominal decompression in extensively burned patients. 2007; 62(6):1365-1369.

56. Cheatham ML, Safcsak K, Fiscina C, et al: Advanced age may limit the survival benefit of open abdominal decompression. 2011; 77(7):856-861.

57. Oda J, Ueyama M, Yamashita K, et al: Effects of escharotomy as abdominal decompression on cardiopulmonary function and visceral perfusion in abdominal compartment syndrome with burn patients. 2005; 59(2):369-374.

58. Ramirez OM, Ruas E, Dellon AL: "Components separation" method for closure of abdominal-wall defects: an anatomic and clinical study. 1990; 86(3):519-526.

59. Palmieri T, Lavrentieva A, Greenhalgh D: An assessment of acute kidney injury with modified RIFLE criteria in pediatric patients with severe burns. 2009; 35(12):21252129. 
60. Soltani A, Karsidag S, Garner W: A ten-year experience with hemodialysis in burn patients at Los Angeles County + USC Medical Center. 2009; 30(5):832-835.

61. Mosier MJ, Pham TN, Klein MB, et al: Early enteral nutrition in burns: compliance with guidelines and associated outcomes in a multicenter study. 2011; 32(1):104-109.

62. Yenikomshian H, Reiss M, Nabavian R, et al: Gastric feedings effectively prophylax against upper gastrointestinal hemorrhage in burn patients. 2011; 32(2):263-268.

63. Sanchez MS, Herrero E, Asensio MJ, et al: Intra-abdominal hypertension (IAH) and abdominal compartment syndrome (ACS) in critically burn patients. 2009; 35((2009)):S46.

64. Malbrain ML, Jacobs A, De laet I, et al: Incidence and prognosis of intra-abdominal hypertension and abdominal compartment syndrome in severely burned patients. 2010; 36((2010)):S375. 